\title{
Time-domain THz spectroscopy reveals coupled protein-hydration dielectric response in solutions of native and fibrils of human lyso-
} zyme

\author{
Fabio Novelli', Saeideh Ostovar Pour ${ }^{2}$, Jonathan Tollerud ${ }^{1}$, Ashkan Roozbeh', Dominique R. T. \\ Appadoo3 $^{3}$ Ewan W. Blanch², Jeffrey A. Davis ${ }^{1, *}$ \\ ${ }^{1}$ Centre for Quantum and Optical Science, Swinburne University of Technology, Victoria 3122, Australia \\ ${ }^{2}$ School of Science, RMIT University, GPO Box 2476, Melbourne, Victoria 3001, Australia \\ ${ }^{3}$ Australian Synchrotron, 8oo Blackburn Road, Clayton, 3168 VIC, Australia
}

\begin{abstract}
Here we reveal details of the interaction between human lysozyme proteins, both native and fibrils, and their water environment by intense terahertz time domain spectroscopy. With the aid of a rigorous dielectric model, we determine the amplitude and phase of the oscillating dipole induced by the THz field in the volume containing the protein and its hydration water. At low concentrations, the amplitude of this induced dipolar response decreases with increasing concentration. Beyond a certain threshold, marking the onset of the interactions between the extended hydration shells, the amplitude remains fixed but the phase of the induced dipolar response, which is initially in phase with the applied $\mathrm{THz}$ field, begins to change. The changes observed in the $\mathrm{THz}$ response reveal protein-protein interactions mediated by extended hydration layers, which may control fibril formation and may have an important role in chemical recognition phenomena.
\end{abstract}

Proteins are the most versatile macromolecules of the cell and the ability to maintain their native, functional state is fundamental to biological activity. A great number of mammalian disorders have some linkage with protein misfolding and aggregation. Amyloid fibril related disorders[1-4], for example, are associated with the unwanted filamentous aggregation of particular proteins or peptides. Human lysozyme is a good model system that has been used for decades to study amyloid fibrillogenisis. The common features of amyloidogenic proteins are $\beta$-sheet formation and fibrillar morphology[5], however, the detailed mechanism of $\beta$-sheet formation and the molecular level properties of fibrils are not yet understood despite being subjects of great interest[6-8].

The importance of the dielectric solvated environment, particularly of water molecules in mediating the secondary and tertiary structure of proteins, interactions between proteins $[9,10]$, and conformational changes that lead to aggregation is widely appreciated[11-21], but poorly characterized.

Light at terahertz (THz) frequencies (o.1-10 $\mathrm{THz}$ or 3,00o-30 $\mu \mathrm{m}$ ) is strongly absorbed by water and provides a very sensitive probe of any perturbations to the hydrogen bonding network[22,23]. At the interface of a macromolecule in solution several things typically happen: the water molecules close to the surface will be strongly influenced and their dynamics retarded by charges on the surface of the macromolecule, leading to changes in the THz absorp- tion spectrum[24,25]. The influence of protein-water interactions also extends beyond these tightly bound water molecules[26-29], causing changes to the hydrogen bonding network and thus to the contribution of both single water molecules and the larger domains to the THz absorption spectrum. Details of these changes, and how far these effects propagate from the surface of the macromolecule, remain open questions. Techniques based on NMR[30] and neutron or X-ray scattering[31] probe only the tightly bound water molecules, indicating that a sub-monolayer of water molecules is formed around each protein at near physiological conditions. Similar conclusions are drawn from terahertz spectroscopy when films[32-34] or relatively large protein concentrations are studied $(\geq 60 \mathrm{mg} / \mathrm{ml}$ for hen egg white lysozyme[35]). However, when lower concentrations are used, terahertz techniques suggest that the population of hydration water is much larger and extends over multiple layers. Extended hydration shells, where the effect of the protein on the water molecules is evident, have been reported to vary between $15 \AA$ and $25 \AA$ from the solute surface for different proteins[25-29]. While these works are based on the simplistic assumption that the absorption coefficient of a complex system can always be written as the weighted sum of the absorption of its components[35], here we show how determining the complex dielectric response provides a much more informative and reliable assessment of the impact of protein-water interactions on the water environment. Another recent work investigated the full dielectric response of lysozyme films[34]. However, results on films[33,34] cannot be easily 
compared with results on solutions because additional collective modes appear upon film formation[36], and because films typically have much lower water content (compare e.g. the dielectric functions shown in Ref.[34] with the one of bare water in Ref.[23]).

Here we report high-resolution terahertz time-domain measurements of solutions containing native human lysozyme or fibrils down to concentrations as low as $5 \mathrm{mg} / \mathrm{ml}$ (corresponding to $\sim 0.36 \%$ volume fraction) at room temperature. By determination of the complex dielectric function, we are then able to determine the amplitude and phase of the induced dipole in the volume containing the protein and the extended hydration water. Further details of the hydration layer and interactions between proteins are then revealed by tracking the concentration dependence of these values.

We measured the amplitude and the phase of the $\mathrm{THz}$ field transmitted through $0.5 \mathrm{~mm}$ thick lysozyme-water solutions at room temperature. We used large amplitude $\mathrm{THz}$ fields generated by tilted-front optical rectification[37-41] and detected by electro-optical sampling[4244], as described in the Supplementary Information. The associated pulse fluence is lower than $2 \mu \mathrm{J} / \mathrm{cm}^{2}$ and any thermal effects can be disregarded[45]. Human lysozyme was obtained from Sigma Aldrich (Australia). The samples were prepared in milli-Q water with $\mathrm{pH}$ value of $4.8 \mathrm{o}$ for solutions containing native proteins, and with $\mathrm{pH} 2.0$ for solutions containing fibrils. In order to form fibrils of human lysozyme the samples were incubated at $96^{\circ} \mathrm{C}$ for 16 hours in a solution with $\mathrm{pH} 2.0$ [46]. The formation of fibrils following this protocol has previously been verified by AFM, TEM and Thiflavin T assay[46]. Additional details of sample preparation are discussed in the Supplementary Information. The distilled water and the protein-free solutions at $\mathrm{pH} 2.0$ and 4.8 all have, within our resolution, the same optical absorption and refraction properties at the terahertz frequencies investigated in this work. In the following we simply take distilled water as the background for zero protein concentrations. The samples are well shaken before the measurements, and the fields transmitted by a reference and by the filled quartz cell are acquired alternately 10 times for a total acquisition window shorter than 15 minutes per sample.

In Figure 1 we report typical time-domain traces of the $\mathrm{THz}$ pulses transmitted by a reference (Figure 1a) and by $100 \mathrm{mg} / \mathrm{ml}$ solutions of native human lysozyme (blue curve in Figure $1 b$ ) and fibrils (red curve in Figure $1 b$ ). By comparing the blue and red curves in Figure $1 b$ it can be seen that the terahertz response of fibrils and native solutions is distinctly different: the solution containing the fibrils displays lower transmission and is delayed in time. The inset of Figure $\mathrm{ib}$ shows the spectral amplitude of the transmission, compared to the incident spectrum (the dips in the incident $\mathrm{THz}$ spectrum at and above $\sim 1.1 \mathrm{THz}$ are due to absorption by water molecules in the air[47]). The large drop (>20x) in the THz spectral amplitude transmitted by the protein solutions is primarily due to absorption by bulk water. However, by normalizing the transmission of the protein solutions to the transmission through pure water (see Supplementary Figurei(a)), it can be seen that the spectral response of both native lysozyme and fibrils is flat and that the fibrils transmit less than the native lysozyme. These results are confirmed by additional measurements performed at the FAR-IR beamline of the Australian Synchrotron (see Supplementary Figure $1(b, c)$ ).
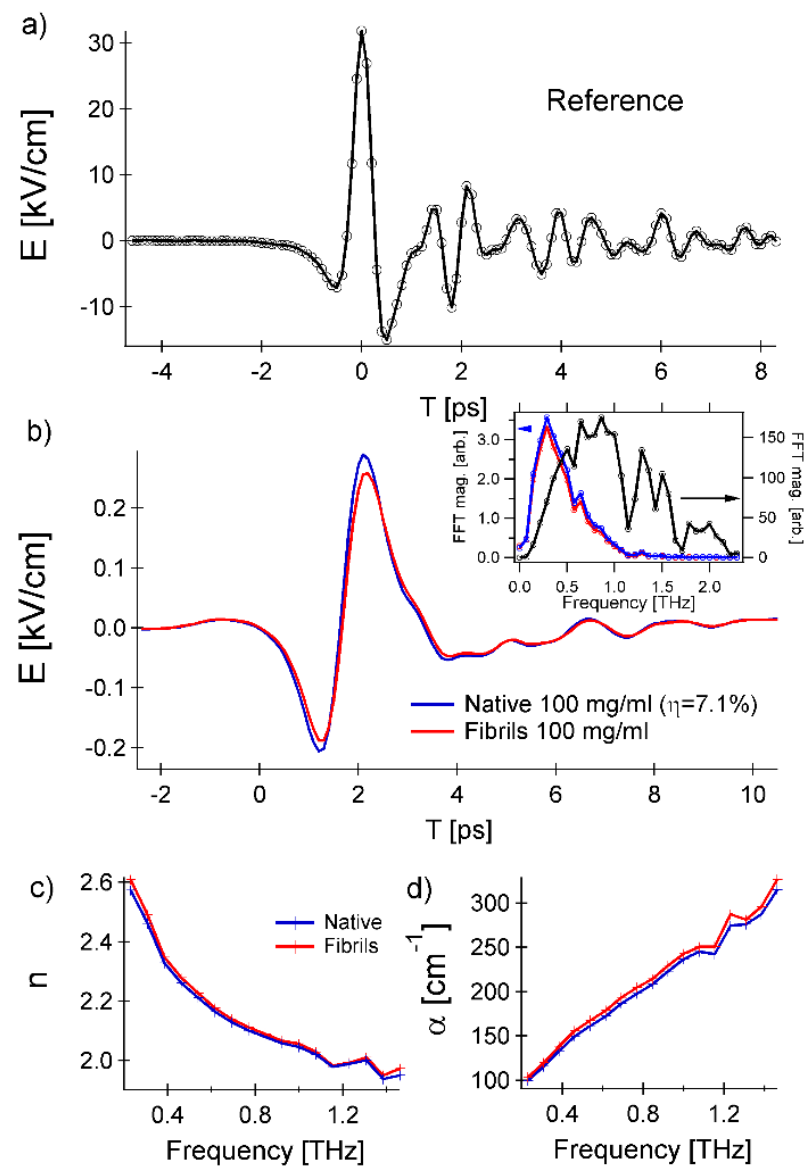

Figure 1. a) Amplitude of the pulsed THz field transmitted through the empty sample holder as a function of the sampling pulse delay. b) Typical THz pulses transmitted by water-based solutions containing $100 \mathrm{mg} / \mathrm{ml}$ native (blue) and fibrils (red). In the inset the magnitude of the Fourier transformations are shown as a function of frequency. c) The index of refraction for the lysozyme solutions and d) The corresponding absorption coefficient.

From the time-domain $\mathrm{THz}$ experiments we calculate the full dielectric properties of the solutions, both the index of refraction (Figure ic) and the absorption coefficient (Figure 1d), assuming only the first Fresnel transmission coefficient is relevant (see Supplementary Information for details). It is important to note that scattering cannot explain the differences observed between fibril and native protein solutions (see Supplementary Information). 
For the following analysis we focus on the average values for the optical properties over the $0.4-0.9 \mathrm{THz}$ range. The average absorption coefficient is shown in Figure 2a for native (blue solid squares) and fibril (red solid squares) lysozyme solutions, together with the expected effect of removing a volume of bulk water equal to the protein volume (black line in Figure 2a). The optical properties depend on the volume fraction, $\eta$, of the proteins in solution. For native lysozyme we measured a variety of solutions with $\eta$ ranging from about $0.36 \%$ to $42.7 \%$ (or, equivalently, from 5 to $600 \mathrm{mg} / \mathrm{ml}$, taking the volume of each protein as a simple sphere with radius $\sim 15.9 \mathrm{~A}[48]$ and assuming ideal behavior [49]). Three distinct regions can be identified in Fig. 2(a) for the native human lysozyme (blue squares): above the solution saturation threshold of $\eta \sim 17.8 \%(250 \mathrm{mg} / \mathrm{ml})$ a strong deviation from water-removal effects is seen, as expected[35]; between $\eta=7.1 \%$ and $17.8 \%$ (100 and 250 $\mathrm{mg} / \mathrm{ml}$ ) a linear decrease is seen, showing reasonable agreement with bulk water-removal effects[35]; and at volume fractions in the range $0.36 \%-3.6 \%(5-50 \mathrm{mg} / \mathrm{ml})$ a flatter region is evident (zoomed in Figure $2 b$ ), which cannot be explained as removal of bulk water[29], but can be described in part by contributions from the water molecules that are affected by the presence of the protein[24,29]. These results are consistent with previous measurement of $\mathrm{THz}$ absorption of lysozyme over different parts of this concentration range[29,35].

The solutions containing fibrils of lysozyme have been studied below the saturation point, between $\eta \sim 0.36 \%(5$ $\mathrm{mg} / \mathrm{ml})$ and $14.2 \%(200 \mathrm{mg} / \mathrm{ml})$. It is evident that the solutions with fibrils display a larger absorption and index of refraction compared to the native lysozyme (see Figure 1 and Figure 2).

In the following we focus on the results obtained below $\eta \sim 3.6 \%(50 \mathrm{mg} / \mathrm{ml})$ where the protein volume is known not to change for different concentrations[49].

To understand the volume-fraction dependent response of the fibril and native human lysozyme solutions shown in Figure 2 we begin by following the approach of Heyden et al.[24]. The starting point is to consider the protein volume as a void. The first order effect is simply the removal of bulk water, which leads to a reduction in the absorption linearly proportional to the volume fraction of the protein in solution (this is the solid black line in Figure $2 a$ and Figure $2 b$ ). However, in a dielectric medium the applied field will also induce a polarization at the interface between the void and the dielectric. If we consider the protein to be a spherical void and water to be a static dielectric medium, Maxwell's equations tell us that the induced field will be in the opposite direction to the applied field, with magnitude given by $\boldsymbol{M}^{M}=-V \boldsymbol{P} \frac{3 \epsilon_{S}}{2 \epsilon_{S}+1}$ where $V$ is the void volume, $\boldsymbol{P}$ is the transverse bulk polarization, and $\epsilon_{S}$ is the dielectric constant of the solvent[24]. Under these assumptions the resultant average dipole moment induced over the solution, and hence the THz absorption, is further reduced.
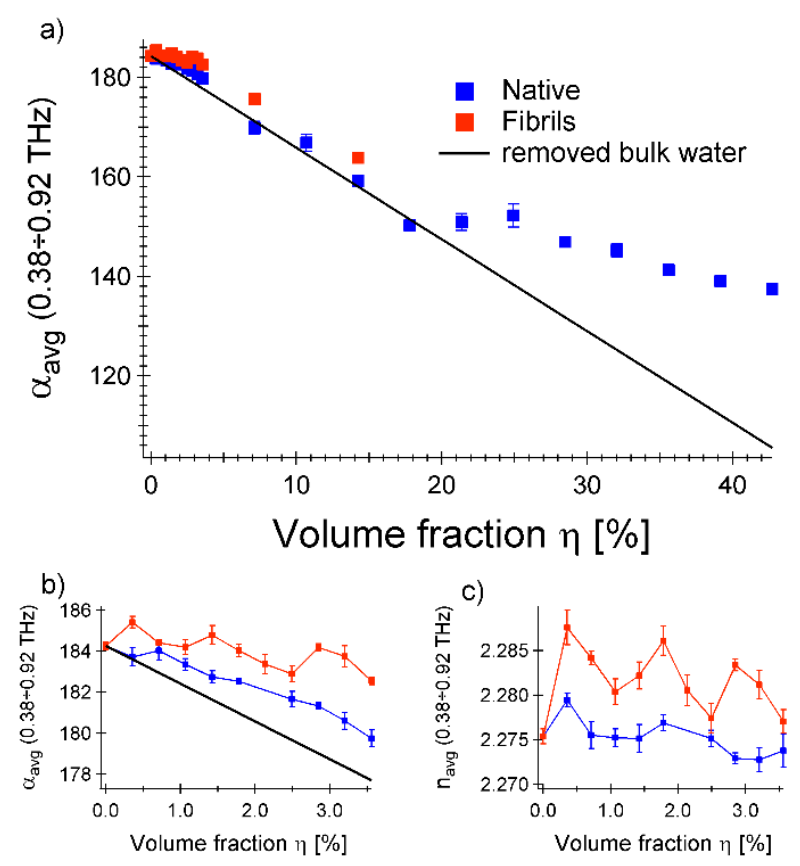

Figure 2. a) The average absorption coefficient in the $0.38-0.92 \mathrm{THz}$ range of the lysozyme solutions is shown versus the volume fraction of lysozyme. b) Same as a) but on an expanded scale for small concentrations or volume fractions. Error bars are \pm 1 standard deviations calculated from 10 measurements (see Supplementary Information). c) Average index of refraction at low concentrations.

Previous measurements of proteins (including lysozyme) in water solutions have, however, shown that rather than the $\mathrm{THz}$ absorption being lower than expected when considering only water removal, the $\mathrm{THz}$ absorption is actually higher[24-29], consistent with the results reported here (Fig. 2).

The absorption is enhanced because the protein is not a void and will typically have some absorption and surface charges, and because the water environment of the protein is not a static dielectric but is able to move and rotate to compensate for an electromagnetic perturbation. It is clear then that the pure Maxwell picture described above does not provide a complete description. Heyden et al.[24] thus defined the scalar quantity " $a$ " relating the actual dipole moment induced in the volume of solution containing one protein and its hydration layers, $\boldsymbol{M}^{\text {int }}$, to the dipole moment predicted in the case of one spherical void in a static dielectric $\boldsymbol{M}^{M}$ :

$$
a=\hat{\boldsymbol{x}} \cdot \frac{\boldsymbol{M}^{\text {int }}}{\boldsymbol{M}^{M}}
$$

where $\hat{\boldsymbol{x}}$ is an unitary vector along the direction of the applied electric field. This can be combined with the contribution from bulk water and its removal to give the complex, frequency-dependent dielectric response of the solution, $\epsilon(\omega)$ : 


$$
\epsilon(\omega)=1+\left[\epsilon_{S}(\omega)-1\right][1-\eta]-\eta\left[a(\omega) \frac{\left[\epsilon_{S}(\omega)-1\right]^{2}}{2 \epsilon_{S}(\omega)+1}\right] \text { Eq.2 }
$$

where $\eta$ is the volume fraction of the protein in solution and $\epsilon_{S}(\omega)$ is the dielectric function of the solvent alone[24]. In the case that $a=1$ the induced dipole will follow the behaviour predicted from Maxwell's electrostatics $\boldsymbol{M}^{\text {int }}=\boldsymbol{M}^{M}$; for $a=0$ the solvent rearrangement will perfectly compensate for the Maxwell dielectric field and the behaviour of the solution can be described simply as removal of bulk water.

In several previous works[28,50] the THz absorption of protein solutions decreases slower than expected for solvent removal, or in some cases even increases as a function of protein concentration[24-27,29]. This corresponds to a negative value of $a$ and an induced dipole, in the volume containing protein and hydration water molecules (i.e. $\left.\boldsymbol{M}^{\text {int }}\right)$, that is parallel with the applied field direction. This is consistent with the absorption behavior observed here; however, we also have the refractive index, which allows a reassessment of Eq.1 and the determination of both amplitude and phase of the induced dipole.

The analysis proposed by Heyden et al.[24], based on Eq. 1 , indicates that only the component of the induced field in phase (negative $a$ ) or $\pi$ out of phase (positive $a$ ) with the applied field is relevant. In general, however, the induced dipole can have any phase relative to the applied field. Determination of the full complex dielectric function, as we obtain here, provides additional constraints that allow us to determine a complex value for $\boldsymbol{a}$ which relates to the amplitude and phase of $\boldsymbol{M}^{\text {int }}$, not simply the projection of the induced-dipole onto the applied field direction. In this context, the scalar product in Eq. 1 is between the induced dipole and the axis of the applied field rather than the oscillating field direction.

We determine the complex valued $\boldsymbol{a}(\boldsymbol{\omega})$ using Eq. 2, the $\alpha$ and $n$ values obtained previously (see Fig.1 and Fig.2), and the measured values for the complex dielectric function of the pure solvent. The amplitude and phase of $\boldsymbol{a}$ are plotted for both the fibrils and native lysozyme in Figure 3 (the lines represent different $\mathrm{THz}$ frequencies while the points correspond to the average values).

For native lysozyme the average amplitude of $\mathbf{a},|\mathbf{a}|$, is initially $1.4 \pm 0.3$ and the phase, $\Phi(\mathbf{a})$, is $-\pi$. This corresponds to an induced field, $\boldsymbol{M}^{\text {int }}$, oscillating in phase with the applied field and having a magnitude consistent with previous reports on a variety of different macromolecules in water solutions (see e.g. Ref.[24] and references therein). A subsequent rapid drop of $|\mathbf{a}|$ as the concentration is increased takes the amplitude down to $0.6 \pm 0.1$, where it remains flat for the rest of the concentration range. From this point on, however, the average phase increases from $-\pi$ at the lowest concentration to $-0.4 \pi$ at the volume fraction of $3.6 \%$.
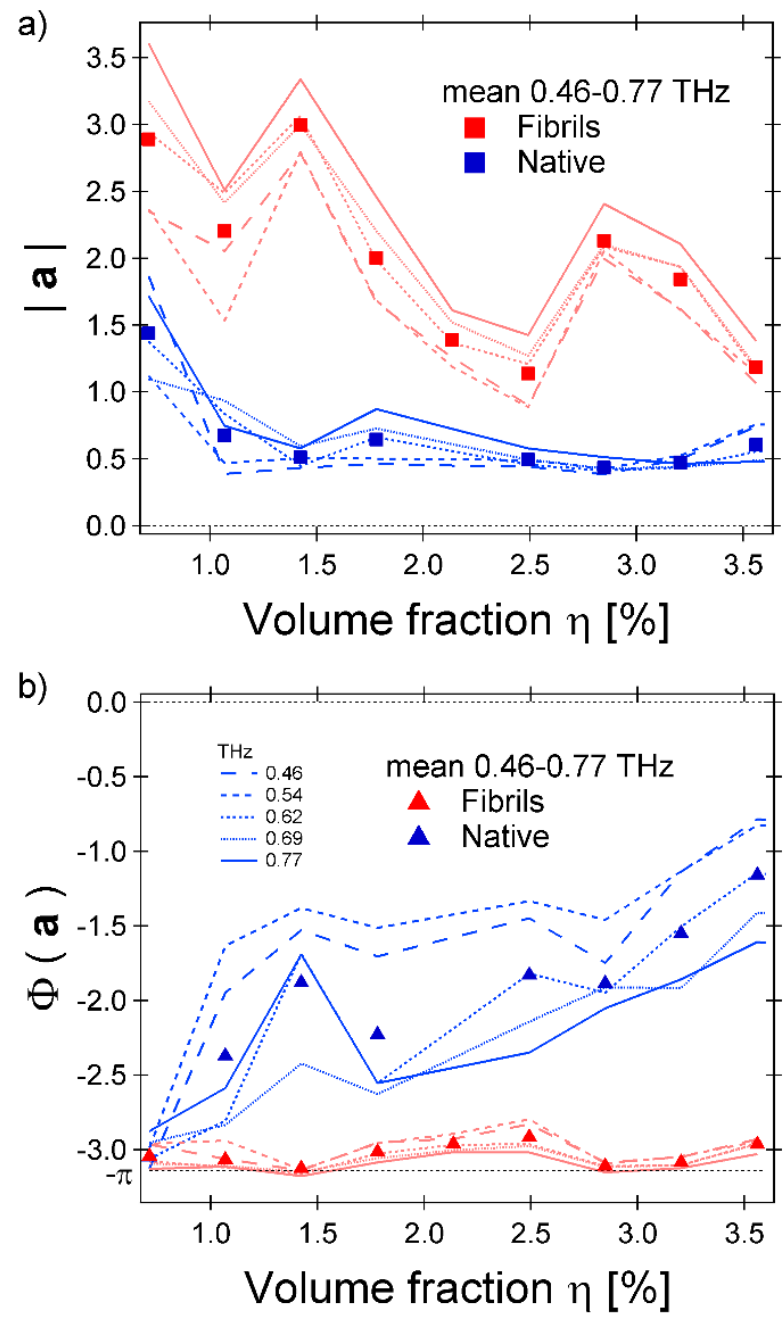

Figure 3. a) Relative amplitude and b) phase of the induced dipole at the protein-solvent boundary versus volume fraction of lysozyme for solutions containing native human lysozyme (blue) and fibrils (red). Squares represent the average response in the $0.46-0.77 \mathrm{THz}$ range, each thin line corresponds to a different frequency from $0.46 \mathrm{THz}$ to $0.77 \mathrm{THz}$ by $\sim 0.07 \mathrm{THz}$ steps (legend inside panel b).

Precise quantitative details of the induced dipole moment at the low concentration limit are determined by the nanoscopic interactions within and between the protein/s and the surrounding water molecules, and requires detailed molecular modelling of the dynamic response, which is beyond the scope of this work. However, as a phenomenological explanation of our results we propose that the two regimes observed correspond to the two cases shown in Figure 4(b). At low concentrations $|\mathbf{a}|$ reduces as the probability of the solvation shells overlapping slightly increases, as proposed by Heyden et al[24]. In this case, most of the proteins and their solvation waters are not interacting with the solvation shells of other proteins. Above the critical concentration, where $|\mathbf{a}|$ remains constant and 
$\Phi($ a) changes, there is significant overlap between the solvation shells of neighboring proteins. In this regime the solvation water is the dominant "type" of water and the strength of the interaction between the solvation waters and the proteins increases as the average distance between them decreases. This then affects the ability of the induced dipole to follow the field, which results in the phase shift observed.
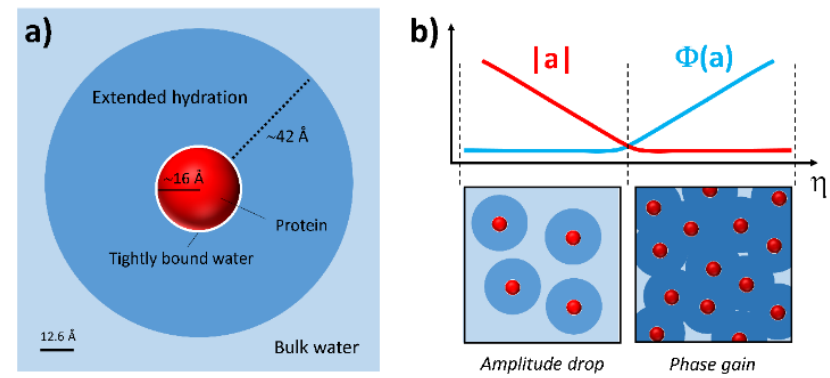

Figure 4. a) Cartoon of a human lysozyme protein (red sphere) in water together with the populations of: i) water molecules tightly-bound to the protein surface (white), ii) extended hydration layers (blue), and iii) unperturbed bulk water (light blue). b) Sketch of the evolution of the modulus and the phase of the induced dipole in a unit volume of solution versus protein concentration. The effect of phase gain at larger concentrations is pictorially represented by darker colors on the bottom right panel.

Simply assuming that all bulk water has been replaced at the point where the trends of both $|\mathbf{a}|$ and $\Phi(\mathbf{a})$ change, (i.e. at $\eta \sim 1 \%$ or $15 \mathrm{mg} / \mathrm{ml}$ for native solutions), and considering a uniform cube lattice of proteins, we estimate[48] an upper limit for the extent of the hydration layer around each native protein. This indicates the hydration layer may extend up to $42 \AA$ from the protein surface for dilute solutions. This value is on the same order of magnitude as previous reports[26-29], and is very similar to the size of the extended hydration layer calculated by molecular dynamics calculations for other protein systems[24].

For the solutions containing fibrils, the plots in Figure 3 for the amplitude (red squares) and phase (red triangles) of $\boldsymbol{a}$ are quite different: the amplitude slowly decreases while the phase remains flat at $-\pi$. This behavior of the fibrils up to $\eta \sim 3.6 \%(50 \mathrm{mg} / \mathrm{ml})$ is equivalent to the behavior of native lysozyme for $\eta<1 \%(<15 \mathrm{mg} / \mathrm{ml})$ and is consistent with the phenomenological model proposed above, whereby the dominant trend is determined by the average distance between macromolecular assemblies. For a given concentration of native lysozyme the separation between fibrils, each originating from many closely spaced lysozyme molecules, is much larger, less hydration water is present, and the hydration layers do not overlap. A precise quantitative comparison, however, is prevented by the large heterogeneity in the fibril size (see Methods in SI).
The other major difference observed in the fibrils is that the initial value for $|\boldsymbol{a}|$ is $2.8 \pm 0.6$, significantly larger than the initial value for native lysozyme. This indicates that the induced dipole, $\boldsymbol{M}^{\text {int }}$, for the fibrils is larger than for the native. Previous work has revealed that in fibrils the lysozyme tertiary structure changes, such that there is a high density of $\beta$-sheets on their surface[51]. This may explain the greater value of $\boldsymbol{M}^{\text {int }}$ because the higher surface charge density from the $\beta$-sheets can cause a greater disruption to the hydrogen-bonding network of water, however, detailed modelling is required to quantitatively compare the two.

Regardless, the demonstrated ability to reveal differences in the $\mathrm{THz}$ response of native proteins and fibrils indicates that terahertz techniques can help to understand the nature of protein-environment interactions and the role they play in stabilizing tertiary structure and driving changes to it[52].

In conclusion, determination of the complex dielectric function of proteins in solution, enabled by time domain THz spectroscopy, allows detailed analysis beyond what is possible from simple absorption measurements. Following this approach we are able to determine both the phase and amplitude of the induced dipole in the volume containing the protein and solvating water. Separating the amplitude and phase of this response reveals that not only can the amplitude of the induced dipole vary as the concentration is changed, but above a concentration threshold so too does the phase of the induced dipole oscillations. While the precise details of the $\mathrm{THz}$ response are determined by dynamic atomistic interactions, we propose a phenomenological model that explains the available data, whereby the phase of the induced dipole in the protein-solvent interaction region begins to vary when there is significant overlap between solvation layers of neighboring proteins. This result suggests that indirect electromagnetic protein-protein interactions could take place if mediated by the extended hydration layers surrounding each protein which might also be of great relevance for chemical recognition $[52,53]$.

\section{AUTHOR INFORMATION NOTES}

\section{Corresponding Author}

*JDavis@swin.edu.au

\section{ACKNOWLEDGMENT}

We thank the Australian Research Council for funding (DP130101690). FN acknowledges funding from Swinburne University via the Early/Interrupted research career scheme 2014. This work has been partially done at the Australian Synchrotron during beamtime AS153/FIR/9692 at the FAR-IR beamline.

\section{REFERENCES}

[1] Baumeister, R.; Eimer, S. Amyloid Aggregates Presenilins, and Alzheimers Disease. Angewandte Chemie International Edition 1998, 37 (21), 2978-82. 
[2] Rhoades, E.; Agarwal, J.; Gafni, A. Aggregation of an Amyloidogenic Fragment of Human Islet Amyloid Polypeptide. Biochimica Et Biophysica Acta (BBA) - Protein Structure and Molecular Enzymology 2000, 1476 (2), 230-38.

[3] DiFiglia, M.; Sapp, E.; Chase, K.O.; Davies, S.W.; Bates, G.P.; Vonsattel, J.P.; Aronin, N. Aggregation of Huntingtin in Neuronal Intranuclear Inclusions and Dystrophic Neurites in Brain. Science 1997, 277 (5334), 1990-93.

[4] Prusiner, S.B. Molecular Biology of Prion Diseases. Science 1991, 252 (5012), 1515-22.

[5] Campioni, S.; Mannini, B.; Zampagni, M.; Pensalfini, A.; Parrini, C.; Evangelisti, E.; Relini, A.; Stefani, M.; Dobson, C.M.; Cecchi, C.; Chiti, F. A Causative Link between the Structure of Aberrant Protein Oligomers and Their Toxicity. Nature Chemical Biology 2010, 6 (2), 140-47.

[6] Liu, R.; He, M.; Su, R.; Yu, Y.; Qi, W.; He, Z. Insulin Amyloid Fibrillation Studied by Terahertz Spectroscopy and Other Biophysical Methods. Biochemical and Biophysical Research Communications 2010, 391 (1), 862-67.

[7] Png, G.M.; Falconer, R.J.; Abbott, D. Tracking Aggregation and Fibrillation of Globular Proteins Using Terahertz and Far-Infrared Spectroscopies. IEEE Transactions on Terahertz Science and Technology 2016, 6 (1), 45-53.

[8] Zakaria, H.A.; Fischer, B.M.; Bradley, A.P.; Jones, I.; Abbott, D.; Middelberg, A.P.J.; Falconer, R.J. Low-Frequency Spectroscopic Analysis of Monomeric and Fibrillar Lysozyme. Applied Spectroscopy 2011, 65 (3), 260-64.

[9] Ebbinghaus, S.; Meister, K.; Born, B.; DeVries, A.L.; Gruebele, M.; Havenith, M. Antifreeze Glycoprotein Activity Correlates with Long-Range Protein-Water Dynamics J. Am. Chem. Soc. 2010, 132 (9), 12210-12211.

[10] Xu, Y.; Havenith, M. Watching low-frequency vibrations of water in biomolecular recognition by THz spectroscopy The Journal of Chemical Physics 2015, 143, 170901.

[11] Cho, C.H.; Singh, S.; Robinson, G.W. Liquid Water and Biological Systems: the Most Important Problem in Science That Hardly Anyone Wants to See Solved. Faraday Disc.1996, 103, 1927.

[12] Roberts, C.J. Kinetics of Irreversible Protein Aggregation: Analysis of Extended Lumry-Eyring Models and Implications for Predicting Protein Shelf Life. The Journal of Physical Chemistry $B$ 2003, 107 (5), 1194-1207.

[13] Tsai, C.J.; Maizel, J.V.; Nussinov, R. The Hydrophobic Effect: A New Insight from Cold Denaturation and a Two-State Water Structure. Critical Reviews in Biochemistry and Molecular Biology 2002, 37 (2), 55-69.

[14] Ben-Amotz, D.; Underwood, R. Unraveling Water's Entropic Mysteries: A Unified View of Nonpolar Polar, and Ionic Hydration. Accounts of Chemical Research 2008, 41 (8), 957-67.

[15] Disalvo, E.A.; Lairion, F.; Martini, F.; Tymczyszyn, E.; Frías, M.; Almaleck, H.; Gordillo, G.J. Structural and Functional Properties of Hydration and Confined Water in Membrane Interfaces. Biochimica Et Biophysica Acta (BBA) - Biomembranes 2008, 1778 (12), 2655-70.

[16] Gallo, P.; Rovere, M. Structural Properties and Liquid Spinodal of Water Confined in a Hydrophobic Environment. Physical Review E 2007, 76 (6), o61202.

[17] Szyperski, T.; Mills, J.L. NMR-Based Structural Biology of Proteins in Supercooled Water. J Struct Funct Genomics 2011, 12 (1), 1-7.

[18] Prabhu, N.; Sharp, K. ProteinSolvent Interactions. ChemInform 2006, 37 (30), 200630298.

[19] Dill, K.A.; Truskett, T.M.; Vlachy, V.; Hribar-Lee, B. Modeling Water the Hydrophobic Effect, and Ion Solvation. Annual
Review of Biophysics and Biomolecular Structure 2005, 34 (1), 17399.

[20] Teeter, M.M. Water-Protein Interactions: Theory and Experiment. Annu. Rev. Biophys. Biophys. Chem. 1991, 20 (1), 577600.

[21] Schnepf, M.I. Protein-Water Interactions. In Biochemistry of Food Proteins, Hudson, B.J.P., Eds.; Springer: 1992, 1-33.

[22] Falconer, R.J.; Markelz, A.G. Terahertz Spectroscopic Analysis of Peptides and Proteins. Journal of Infrared Millimeter, and Terahertz Waves 2012, 33 (10), 973-88.

[23] Vinh, N.Q.; Sherwin, M.S.; Allen, S.J.; George, D.K.; Rahmani, A.J.; Plaxco, K.W. High-precision gigahertz-to-terahertz spectroscopy of aqueous salt solutions as a probe of the femtosecond-to-picosecond dynamics of liquid water. The Journal of Chemical Physics 2015, 142, 164502.

[24] Heyden, M.; Tobias, D.J.; Matyushov, D.V. Terahertz Absorption of Dilute Aqueous Solutions. The Journal of Chemical Physics 2012, 137 (23), 235103.

[25] Xu, J.; Plaxco, K.W.; Allen, S.J. Collective Dynamics of Lysozyme in Water: Terahertz Absorption Spectroscopy and Comparison with Theory. The Journal of Physical Chemistry B 2oo6, $110,24255$.

[26] Ebbinghaus, S.; Kim, S.J.; Heyden, M.; Yu, X.; Heugen, U.; Gruebele, M.; Leitner, D.M.; Havenith, M. An Extended Dynamical Hydration Shell around Proteins. Proceedings of the National Academy of Sciences 2007, 104 (52); 20749-52.

[27] Born, B.; Kim, S.J.; Ebbinghaus, S.; Gruebele, M.; Havenith, $M$. The terahertz dance of water with the proteins: the effect of protein flexibility on the dynamical hydration shell of ubiquitin. Faraday Discuss. 2009, 141, 161-173.

[28] Bye, J.W.; Meliga, S.; Ferachou, D.; Cinque, G.; Zeitler, J.A.; Falconer, R.J. Analysis of the Hydration Water around Bovine Serum Albumin Using Terahertz Coherent Synchrotron Radiation. J. Phys. Chem. A 2014, 118 (1), 83-88.

[29] Sushko, O.; Dubrovka, R.; Donnan, R.S. Sub-Terahertz Spectroscopy Reveals That Proteins Influence the Properties of Water at Greater Distances than Previously Detected. The Journal of Chemical Physics 2015, 142 (5), o55101.

[30] Kuntz, I.D. Hydration of Macromolecules. III. Hydration of Polypeptides. J. Am. Chem. Soc. 1971, 93 (2), 514-16.

[31] Svergun, D.I.; Richard, S.; Koch, M.H.J.; Sayers, Z.; Kuprin, S.; Zaccai, G. Protein Hydration in Solution: Experimental Observation by $\mathrm{x}$-Ray and Neutron Scattering. Proceedings of the National Academy of Sciences 1998, 95 (5), 2267-72.

[32] Knab, J.; Chen, J.Y.; Markelz, A. Hydration Dependence of Conformational Dielectric Relaxation of Lysozyme. Biophysical Journal 2oo6, 90 (7), 2576-81.

[33] Woods, K.N. Solvent-induced backbone fluctuations and the collective librational dynamics of lysozyme studied by terahertz spectroscopy. Physical Review E 2010, 81, 031915.

[34] Yamamoto, N.; Ohta, K.; Tamura, A.; Tominaga, K. Broadband Dielectric Spectroscopy on Lysozyme in the Sub-Gigahertz to Terahertz Frequency Regions: E ff ects of Hydration and Thermal Excitation. The Journal of Physical Chemistry B 2016, 120, 4743.

[35] Vinh, N.Q.; Allen, S.J.; Plaxco, K.W. Dielectric Spectroscopy of Proteins as a Quantitative Experimental Test of Computational Models of Their Low-Frequency Harmonic Motions. J. Am. Chem. Soc. 2011, 133 (23), 8942-47.

[36] Laman, N.; Harsha, S.S.; Grischkowsky, D.; Melinger, J.S. High-Resolution Waveguide THz Spectroscopy of Biological Molecules. Biophysical Journal 2008, 94, 1010.

[37] Hebling, J.; Yeh, K.L.; Hoffmann, M.C.; Bartal, B.; Nelson, K.A. Generation of High-Power Terahertz Pulses by Tilted-Pulse- 
Front Excitation and Their Application Possibilities. Journal of the Optical Society of America B 2008, 25 (7), B6.

[38] Jewariya, M.; Nagai, M.; Tanaka, K. Enhancement of Terahertz Wave Generation by Cascaded $\chi^{3}$ Processes in $\mathrm{LiNbO}_{3}$. Journal of the Optical Society of America B 2009, 26 (9), A1o1.

[39] Blanchard, F.; Sharma, G.; Razzari, L.; Ropagnol, X.; Bandulet, H.C.; Vidal, F.; Morandotti, R.; Kieffer, J.C.; Ozaki, T.; Tiedje, H.; Haugen, H.; Reid, M.; Hegmann, F. Generation of Intense Terahertz Radiation via Optical Methods. IEEE J. Select. Topics Quantum Electron. 2011, 17 (1), 5-16.

[40] Fülöp, J.A.; Pálfalvi, L.; Almási, G.; Hebling, J. Design of High-Energy Terahertz Sources Based on Optical Rectification. Opt. Express 2010, 18 (12), 12311.

[41] Hirori, H.; Tanaka, K. Single-Cycle Terahertz Pulses with Amplitudes Exceeding $1 \mathrm{MV} / \mathrm{Cm}$ Generated by Optical Rectification in $\mathrm{LiNbO}_{3}$ and Applications to Nonlinear Optics. Proc. SPIE 2012, 8240 .

[42] Gallot, G.; Zhang, J.; McGowan, R.W.; Jeon, T.I.; Grischkowsky, D. Measurements of the THz Absorption and Dispersion of ZnTe and Their Relevance to the Electro-Optic Detection of THz Radiation. Appl. Phys. Lett. 1999, 74 (23), 3450.

[43] Wu, Q.; Zhang, X.C. Ultrafast Electro-Optic Field Sensors. Appl. Phys. Lett. 1996, 68 (12), 1604.

[44] Casalbuoni, S.; Schlarb, H.; Schmidt, B.; Steffen, B.; Schmuser, P.; Winter, A. Numerical Studies on the Electro-Optic Sampling of Relativistic Electron Bunches. Proceedings of the 2005 Particle Accelerator Conference 2005, 1591367.

[45] Kristensen, T.T.; Withayachumnankul, W.; Jepsen, P.U.; Abbott, D. Modeling Terahertz Heating Effects on Water. Opt. Express 2010, 18 (5), 4727 .

[46] Krebs, M.R.H.; Wilkins, D.K.; Chung, E.W.; Pitkeathly, M.C.; Chamberlain, A.K.; Zurdo, J.; Robinson, C.V.; Dobson, C.M. Formation and seeding of amyloid fibrils from wild-type hen lysozyme and a peptide fragment from the $\beta$-domain Journal of $\mathrm{Mo}$ lecular Biology 2000, 300 (3), 541-549.

[47] Slocum, D.M.; Slingerland, E.J.; Giles, R.H.; Goyette, T.M. Atmospheric Absorption of Terahertz Radiation and Water Vapor Continuum Effects. Journal of Quantitative Spectroscopy and Radiative Transfer 2013, 127, 49-63.

[48] Lee, B. Calculation of Volume Fluctuation for Globular Protein Models. Proceedings of the National Academy of Sciences 1983, 8 o (2), 622-26.

[49] Fredericks, W.J.; Hammonds, M.C.; Howard, S.B.; Rosenberg, F. Density, thermal expansivity, viscosity and refractive index of lysozyme solutions at crystal growth concentrations. Journal of Crystal Growth 1994, 141, 183.

[5o] Nieuhaus, G.; Heyden, M.; Schmidt, D.A.; Havenith, M. Exploring Hydrophobicity by THz Absorption Spectroscopy of Solvated Amino Acids. Faraday Discuss. 2o11, 150, 193.

[51] Greenwald, J.; Riek, R. Biology of amyloid: structure, function, and regulation. Structure 2010, 18 (10), 1244-1260.

[52] Fichou, Y.; Schirò, G.; Gallat, F.X.; Laguri, C.; Moulin, M.; Combet, J.; Zamponi, M.; Härtlein, M.; Picart, C.; Mossou, E.; Lortat-Jacob, H.; Colletier, J.P.; Tobias, D.J.; Weik, M. Hydration Water Mobility Is Enhanced around Tau Amyloid Fibers. Proceedings of the National Academy of Sciences 2015, 112 (20), 6365-70.

[53] Heyden, M.; Bründermann, E.; Heugen, U.; Nie, G.; Leitner, D.M.; Havenith, M. Long-Range Influence of Carbohydrates on the Solvation Dynamics of Water: Answers from Terahertz Absorption Measurements and Molecular Modeling Simulations. J. Am. Chem. Soc. 2008, 130 (17), 5773-79. 